\title{
Holographic Fidelity Susceptibility
}

\author{
Mohsen Alishahiha ${ }^{a}$ and Amin Faraji Astaneh ${ }^{b}$ \\ ${ }^{a}$ School of Physics, ${ }^{b}$ School of Particles and Accelerators, \\ Institute for Research in Fundamental Sciences (IPM) \\ P.O. Box 19395-5531, Tehran, Iran \\ email: alishah@ipm.ir, faraji@ipm.ir
}

\begin{abstract}
For a field theory with a gravitational dual, we study holographic fidelity susceptibility for two states related by a deformation of a relevant operator. To do so, we study back reaction of a massive scalar field on the asymptotic behavior of the metric in an Einstein gravity coupled to a massive scalar field. Identifying the two states, holographically, with the original and back reacted geometries, the corresponding holographic fidelity susceptibility is given by the difference of the volume of an extremal time slice evaluated on the original and back reacted geometries.
\end{abstract}

\section{INTRODUCTION}

Recent progress on black hole physics has opened up a possibility to make a connection between quantum information theory and back hole physics (see [1] and its citations). Most of our understanding on this subject is due to the gauge/gravity duality. In particular holographic entanglement entropy [2] may be thought of as an example which has made this connection more concrete. Computational complexity is another example [3].

More recently, motivated by complexity/volume duality it was proposed that the volume of a co-dimension one time slice may be related to the fidelity appearing in the literature of quantum information theory [4]. Fidelity is a quantity that measures similarity or distance between two states [5]. When both states under consideration are pure it is given by the inner product of two states. To be more precise, let us start with a quantum pure state $|\Psi(\lambda)\rangle$ in the Hilbert of a quantum system with $\lambda$ being a tunable parameter of the model. Then for two pure states parametrized by $\lambda_{1}$ and $\lambda_{2}$ the fidelity is

$$
\mathcal{F}=\left|\left\langle\Psi\left(\lambda_{2}\right) \mid \Psi\left(\lambda_{1}\right)\right\rangle\right|
$$

which for small $\delta \lambda=\lambda_{2}-\lambda_{1}$ it can be expanded as follows

$$
\mathcal{F}=1-G_{\lambda}(\delta \lambda)^{2}+\mathcal{O}\left(\delta \lambda^{3}\right)
$$

where $G_{\lambda}$ is fidelity susceptibility (see [6] for a review). If one perturbes the first state by an operator with dimension $\Delta$, up to a constant of order of one factor, the corresponding fidelity susceptibility will be scaled as (see e.g. [4])

$$
G_{\Delta} \sim \frac{L^{2 \Delta-d-2}}{\epsilon^{2 \Delta-d-2}}
$$

where $\epsilon$ is a UV cut off and $L$ is a length scale of the model.

It is proposed that when the system is deformed by an exact marginal operator, $\Delta=d+1$, the holographic dual of the fidelity susceptibility is given by the volume of a certain co-dimension one hypersurface in an AdS geometry (see [7] for reduced fidelity).
The aim of this letter is to make this correspondence more precise and in particular to extend it for a case where the system is deformed by a relevant operator. Holographically, we will do that by studying the back reaction of a massive scalar field on the metric in an Einstein gravity coupled to a massive scaler field. We should admit that most of the materials we need have been already presented rather extensively in the literature as we shall review in section two. The main contribution of the present letter is to come up with a new application of the results given in section three.

\section{REVIEW OF BACKGROUND GEOMETRY}

In this section we shall present the asymptotic behaviors of the metric and a scalar field for a gravitational theory whose action is given by

$$
I=-\frac{1}{16 \pi G_{N}} \int d^{d+2} x \sqrt{-G}\left(R-\frac{1}{2}(\partial \Phi)^{2}-V(\Phi)\right),
$$

where $V(\Phi)=-\frac{d(d+1)}{L^{2}}+\frac{1}{2} m^{2} \Phi^{2}$ and $L$ is a length scale of the model. An asymptotic AdS solution of the model taking into account the back reaction of the scalar field may be given by [8]

$$
\begin{gathered}
d s^{2}=G_{\mu \nu} d X^{\mu} d X^{\nu}=\frac{L^{2}}{4 \rho^{2}} d \rho^{2}+\frac{1}{\rho} g_{i j}\left(x^{i}, \rho\right) d x^{i} d x^{j}, \\
\Phi(x, \rho)=\rho^{\alpha / 2}\left(\phi_{0}\left(x^{i}\right)+\rho \phi_{1}\left(x^{i}\right)+\cdots\right) .
\end{gathered}
$$

with $i, j=0,1 \cdots, d$ and

$$
\begin{aligned}
g_{i j}\left(x^{i}, \rho\right)= & g_{i j}^{(0)}\left(x^{i}\right)+\rho g_{i j}^{(1)}\left(x^{i}\right)+\cdots \\
& +\rho^{\alpha}\left(g_{i j}^{(\alpha)}\left(x^{i}\right)+\rho g_{i j}^{(\alpha+1)}\left(x^{i}\right)+\cdots\right) .
\end{aligned}
$$

Here $\alpha=(d+1) / 2-\sqrt{(d+1)^{2} / 4+m^{2} L^{2}}$ is related to the dimension of an operator which is dual to the scalar field; $\Delta=d+1-\alpha$. We shall consider relevant operator; $0 \leq \alpha<(d+1) / 2$. Note that the case of $\alpha=(d+1) / 2$ is excluded due to that fact that in this case $g_{i j}^{\alpha}$ cannot be fixed by perturbative expansion around $\rho=0$. Solving the corresponding equations of motion order by order in 
power of $\rho$ near the boundary $(\rho \rightarrow 0)$ one may find all needed functions appearing in the above expressions in terms of the sources $g_{i j}^{(0)}\left(x^{i}\right)$ and $\phi_{0}\left(x^{i}\right)$ (for $\left.d>1\right)$ []

$$
\begin{aligned}
& g_{i j}^{(1)}=-\frac{L^{2}}{d-1}\left(R_{i j}^{(0)}-\frac{1}{2 d} R^{(0)} g_{i j}^{(0)}\right), \quad g_{i j}^{(\alpha)}=-\frac{\phi_{0}^{2}}{4 d} g_{i j}^{(0)}, \\
& g_{i j}^{(\alpha+1)}=c_{1} \phi_{0}^{2} R_{i j}^{(0)}+c_{2} \phi_{0}^{2} R^{(0)} g_{i j}^{(0)}+c_{3} \partial_{i} \phi_{0} \partial_{j} \phi_{0} \\
& +c_{4} \nabla_{i} \partial_{j} \phi_{0}^{2}+c_{5} g_{i j}^{(0)} \square \phi_{0}^{2}+c_{6} g_{i j}^{(0)}\left(\partial \phi_{0}\right)^{2},
\end{aligned}
$$

where

$$
\begin{array}{ll}
c_{1}=-\alpha \frac{b(\alpha+2)+2(\alpha+1) a}{(d-1)(\alpha+1)} & c_{2}=-\frac{1}{2 d}\left(c_{1}+2 \alpha c_{5}\right), \\
c_{3}=-\frac{2 \alpha+3}{\alpha+1} b-\frac{2(2 \alpha+1)}{\alpha} a & c_{4}=\frac{b}{2}+a, \\
c_{5}=-\frac{2 \alpha d}{(\alpha+1)(d-2 \alpha-1)}, c_{6}=\frac{4(\alpha-1) d}{(\alpha+1)(d-2 \alpha-1)} .
\end{array}
$$

with $a=\alpha c_{6} L^{2}-c_{5}(d-4 \alpha-1) L^{2}$ and $b=-\frac{L^{2}}{4 d}$. Moreover one finds [9]

$$
\phi_{1}=\frac{L^{2}}{2(d-2 \alpha-1)}\left(\square \phi_{0}-\frac{1}{2 d} R^{(0)} \phi_{0}\right) .
$$

Here for simplicity we have dropped the explicit dependence of functions on $x^{i}$. Note also that the term corresponding to $c_{6}$ was missed in [9], see however [10]. To the best of our knowledge no body has explicitly computed $c_{5}$ and $c_{6}$, previously.

\section{CHANGE OF VOLUME AND FIDELITY}

In this section we would like to study the leading divergent term of the volume of an extremal co-dimension one hypersurface in the back reacted geometry presented in the previous section. To proceed, consider a time slice on the boundary whose extension to the bulk is the desired extremal hypersurface. The profile of the corresponding hypersurface in the bulk is given by $X^{\mu}\left(Y^{A}\right)$ with $Y^{A}=\left(y^{a}, \sigma\right)$, and $a=1, \cdots, d$ are coordinates of it. The induced metric on the hypersurface is

$$
h_{A B}\left[Y^{A}\right]=\partial_{A} X^{\mu} \partial_{B} X^{\nu} G_{\mu \nu}[X] .
$$

Following [4, 7] one is interested in the volume of the corresponding hypersurface (see also [11])

$$
\mathcal{C}=\frac{1}{8 \pi G_{N} L} \int d^{d+1} Y \sqrt{\operatorname{det} h_{A B}}
$$

Extremizing the volume one arrives at

$$
\partial^{A} \partial_{A} X^{\mu}+\Gamma_{\alpha \beta}^{\mu} \partial^{A} X^{\alpha} \partial_{A} X^{\beta}-h^{A B} \gamma_{A B}^{C} \partial_{C} X^{\mu}=0
$$

that is indeed the trace of the Gauss-Weingarten equation. It might be thought of as equations of motion for
$X^{\mu}$. Here $\Gamma_{\alpha \beta}^{\mu}$ and $\gamma_{A B}^{C}$ are connections constructed from metrics $G_{\mu \nu}$ and $h_{A B}$, respectively.

It is useful to take a gauge in which $\sigma=\rho$ and $h_{a \rho}=0$. In this gauge one may consider the following expansion for the profile of the extremal surface [12]

$$
X^{i}\left(\rho, y^{a}\right)=X^{(0) i}\left(y^{a}\right)+\rho X^{(1) i}\left(y^{a}\right)+\cdots .
$$

From the equation (12) one has

$$
X^{(1) i}=\frac{L^{2} K}{2 d} n^{i},
$$

where $K$ is the trace of the extrinsic curvature of the time slice. By making use of equations (10) and (14) it is straightforward to find the asymptotic expansion of the induced metric as follows

$$
\begin{aligned}
& h_{\rho \rho}=\frac{L^{2}}{4 \rho^{2}}\left(1+\rho h_{\rho \rho}^{(1)}+\rho^{\alpha+1} h_{\rho \rho}^{(\alpha+1)}\right)+\cdots, \\
& h_{a b}=\frac{1}{\rho}\left[h_{a b}^{(0)}+\rho h_{a b}^{(1)}+\rho^{\alpha}\left(h_{a b}^{(\alpha)}+\rho h_{a b}^{(\alpha+1)}\right)\right]+\cdots,
\end{aligned}
$$

where $h_{a b}^{(\alpha)}=g_{a b}^{(\alpha)}$ and

$$
\begin{array}{ll}
h_{\rho \rho}^{(1)}=-\frac{L^{2} K^{2}}{d^{2}}, & h_{a b}^{(1)}=g_{a b}^{(1)}+\frac{L^{2} K}{d} K_{a b} \\
h_{\rho \rho}^{(\alpha+1)}=\frac{L^{2} K^{2} \phi_{0}^{2}}{4 d^{3}}, & h_{a b}^{(\alpha+1)}=g_{a b}^{(\alpha+1)}-\frac{L^{2} \phi_{0}^{2} K}{4 d^{2}} K_{a b} .
\end{array}
$$

Here $g_{a b}^{(n)}=e_{a}^{i} e_{b}^{j} g_{i j}^{(n)}$ is the projection of the boundary metric into the time slice and $e_{a}^{i}=\partial_{a} X^{j}$. Note that all functions are functions of $y_{a}$ 's. Note also that $h_{\rho \rho}^{(1)}$ and $h_{a b}^{(1)}$ have been already computed in [11].

Using the asymptotic expression for the induced metric (15) one finds

$$
\begin{aligned}
\delta \mathcal{C}= & \frac{1}{8 \pi G_{N}} \int d^{d} y d \rho \frac{\sqrt{h^{(0)}}}{4 \rho^{\frac{d+2}{2}-\alpha}}\left[\operatorname{Tr} h_{a b}^{(\alpha)}\right. \\
& +\rho\left(h_{\rho \rho}^{(\alpha+1)}+\operatorname{Tr} h_{a b}^{(\alpha+1)}+\operatorname{Tr}\left(h^{(1)} h^{(\alpha)}\right)_{a b}\right. \\
& \left.\left.+\frac{1}{2} \operatorname{Tr} h_{a b}^{(1)} \operatorname{Tr} h_{a b}^{(\alpha)}+\frac{1}{2} h_{\rho \rho}^{(1)} \operatorname{Tr} h_{a b}^{(\alpha)}\right)\right]+\cdots .
\end{aligned}
$$

Thus, setting a regulator surface at $\rho=\epsilon^{2} / L^{2}$, at the leading order the change of the volume due a relevant operator with dimension $\Delta$ is

$$
\begin{aligned}
& \delta \mathcal{C}_{\Delta}=-\frac{1}{64(2 \Delta-d-2) G_{N}} \frac{L^{2 \Delta-d-2}}{\epsilon^{2 \Delta-d-2}} \int d^{d} y \sqrt{h^{(0)}}\left[\phi_{0}^{2}\right. \\
& +\frac{\epsilon^{2}}{L^{2}}\left(\operatorname{Tr} g_{a b}^{(\alpha+1)}-\frac{\phi_{0}^{2}}{8 d}(d+2) \operatorname{Tr} g_{a b}^{(1)}\right. \\
& \left.\left.-\frac{L^{2} K^{2} \phi_{0}^{2}}{8 d^{3}}\left(d^{2}+3 d-2\right)\right)+\cdots\right] \text {. }
\end{aligned}
$$

For flat time slice and constant $\phi_{0}$ the above expression reads

$$
\delta \mathcal{C}_{\Delta}=-\frac{\phi_{0}^{2} V_{d}}{64(2 \Delta-d-2) G_{N}} \frac{L^{2 \Delta-d-2}}{\epsilon^{2 \Delta-d-2}}
$$


where $V_{d}$ is the volume of time slice on the boundary. This should be compared with equation (3).

It is worth noting that for a deformation by an operator with dimension $\Delta=\frac{d}{2}+1$ one gets logarithmic divergence as follows

$$
\delta \mathcal{C}_{\Delta}=-\frac{\int d^{d} y \sqrt{h^{(0)}} \phi_{0}^{2}}{64 d G_{N}} \log \frac{L}{\epsilon} .
$$

To conclude one may propose that the change of volume of the extremal co-dimension one hypersurface due to the effect of a relevant operator could provide a holographic description for fidelity susceptibility in the dual field theory. It should be noted that the logarithmic behavior for fidelity susceptibility has been also found in the literature [6].

To further justify this proposal it is constructive to extend our discussions for the case where the model has a non-trivial dynamical scaling. Indeed fidelity susceptibility for a deformation caused by an operator with dimension $\Delta$ in a field theory with a dynamical exponent scales as $G_{\Delta} \sim \epsilon^{-2 \Delta+d+2 z}$, where $z$ is the dynamical exponent [6]. It is our aim to get this expression using a holographic model. To proceed, we note that holographic description of field theories with non-trivial exponent may be provided by Lifshitz geometries [13].

$$
d S^{2}=-\frac{d t^{2}}{r^{2 z}}+\frac{d r^{2}}{r^{2}}+\frac{\sum_{i=1}^{d} d x_{i}^{2}}{r^{2}} .
$$

To study holographic fidelity for a deformation caused by a relevant operator in this mode, one may consider a massive scalar field in the above geometry. Using the corresponding equations of motion one finds the asymptotic behavior of the scalar field as follows

$$
\Phi(x, r)=r^{\xi}\left(\phi_{0}+r^{2} \phi_{1}+\cdots\right),
$$

where $\xi=(d+z) / 2-\sqrt{(d+z)^{2} / 4+m^{2}}$ and the dimension of the corresponding dual operator is $\Delta=d+z-\xi$. To find the change of the volume one needs to study back reactions of this scalar field on the metric. To do so, essentially we should go through the procedure we have presented in the previous section for an asymptotic AdS solution. Let us just present the arguments and the results and postpone the details to an extend version of this paper. Actually from the asymptotic form of the scalar field one finds that the corresponding corrections of the back reacted metric start at the order of $\delta g_{i j} \sim r^{2 \xi} g_{i j}^{(\xi)}$. Therefore the leading correction to the induced metric of the extremal hypersurface is $\delta h_{a b} \sim r^{2 \xi} h_{a b}^{(\xi)}$. It is then straightforward to find the leading correction to the change of the volume of the extremal hypersurface associated to the time slice $t=$ constant as follows

$$
\delta \mathcal{C}_{\Delta} \sim \int_{\epsilon} d r d^{d} y \frac{\sqrt{h^{(0)}}}{r^{d+1}} r^{2 \xi} \operatorname{Tr} h_{a b}^{(\xi)} \sim \frac{\int d^{d} y \sqrt{h^{(0)}} \phi_{0}^{2}}{\epsilon^{2 \Delta-d-2 z}},
$$

in agreement with the field theory result $[\underline{6}$. In particular for a marginal operator, $\Delta=d+z$, one gets $\epsilon^{-d}$ that is independent of $z$, as expected.

\section{DISCUSSIONS}

In this paper we have studied holographic fidelity susceptibility associated with a relevant operator with dimension $\Delta$ satisfying $(d+1) / 2<\Delta \leq d+1$. Holographically the corresponding fidelity susceptibility is computed by the change of the volume of an extremal co-dimension one time slice due to the change of the background geometry.

Actually the proposal makes a rough connection between holographic boundary state and the volume of an extremal time slice in the bulk (holographic complexity) as $|\psi(\lambda)\rangle=e^{-\mathcal{C}_{\lambda}}\left(\langle\psi(\lambda)|=e^{\mathcal{C}_{\lambda}}\right)$. Thus one has

$$
\left\langle\psi\left(\lambda_{2}\right) \mid \psi\left(\lambda_{1}\right)\right\rangle=e^{\delta \mathcal{C}_{\Delta}} \sim 1+\delta \mathcal{C}_{\Delta} .
$$

Here we have assumed that two states are related by a relevant operator with dimension $\Delta$. Note also that, as we have seen, $\delta \mathcal{C}_{\Delta}<0$.

It is worth mentioning that although our holographic approach applies for relevant operators with the range of dimensions given above, for $(d+1) / 2<\Delta<(d+2) / 2$ the resultant fidelity susceptibility is not UV divergent. For particular value of $\Delta=(d+2) / 2$ the most divergent term is a logarithmic, in agreement with the field theory result $[6]$.

We have found that the main contribution to the most divergent term is given by $\rho^{\alpha} \operatorname{Tr} g_{a b}^{(\alpha)}$ term with $0 \leq \alpha \leq d / 2$. The lower bound corresponds to the marginal operator and the upper bound to that of logarithmic divergent. Note that in this range our procedure also works for $d=1$ case.

More generally for a $d+1$ dimensional field theory with a dynamical exponent $z$ we have found that holographic fidelity susceptibility is scaled as follows

$$
G_{\Delta} \sim\left\{\begin{array}{cc}
\left(\frac{L}{\epsilon}\right)^{2 \Delta-d-2 z} & 2 \Delta-2 z \neq d, \\
\log \frac{L}{\epsilon} & 2 \Delta-2 z=d .
\end{array}\right.
$$

In this letter our main focus was on the most divergent term in the holographic fidelity susceptibility associated with a relevant operator. In general at subleading order one may also get logarithmic divergent term. In fact the logarithmic divergent term appears when $d$ is even. Moreover, it may also contain a finite term that our asymptotic study cannot capture it. Indeed to find the full expression one requires to have the full back reacted geometry.

So far we have considered cases where two states are related by a deformation caused by a relevant operator. One may also consider a case where the deformation is given by the change of the temperature. In this case it is known that the corresponding fidelity susceptibility is indeed the heat capacity. It is then illustrative to study this case in our framework (see also [14, 15]).

Holographically, a thermal state may be associated to a black hole solution. Therefore to find the corresponding 
fidelity susceptibility one needs to compute the change of the volume of the corresponding extremal co-dimension one time slice when we change an AdS geometry to an AdS black hole. Doing so one finds [4]

$$
\delta \mathcal{C}=b_{d} \frac{V_{d} L^{d} d}{4 G \rho_{H}^{d}},
$$

where $\rho_{H}$ is the radius of horizon and $b_{d}$ is a numerical factor (see Eq 25 of [4]). On the other hand computing the heat capacity of the corresponding black hole one gets (see e.g. [16])

$$
C_{v}=\frac{V_{d} L^{d} d}{4 G \rho_{H}^{d}},
$$

that is equal to the holographic fidelity susceptibility up to an order of one numerical factor $b_{d}$. It is, however, important to note that for $d=1$, one has $b_{1}=0$ which is not compatible with the above picture. Of course this can be understood since a BTZ black hole is locally AdS and therefore the change of volume is zero. Indeed AdS and BTZ black hole can be distinguished from their global structures. An Euclidean BTZ black hole has a topology of a torus and thus using an SL(2) transformation one may compute the volume of a co-dimension one time slice after a Wick rotation by which the variation of the volume leads to $\delta \mathcal{C}=\frac{V_{1} L}{4 G \rho_{H}}$, in agreement with black hole result.

\section{ACKNOWLEDGMENTS}

We would like to thank A. Akhavan, A. Mollabashi, M. R. Mohammadi Mozaffar, A. Naseh, A. Shirzad, M. R. Tanhayi and M. H. Vahidinia for useful discussions. We would also like to thank T. Takayanagi for a correspondence. A.F.A would like to thank the CERN THdepartment for very kind hospitality during working on this project. This work is supported by Iranian National Science Fundation (INSF).
[1] A. Almheiri, D. Marolf, J. Polchinski and J. Sully, "Black Holes: Complementarity or Firewalls?," JHEP 1302, 062 (2013) arXiv:1207.3123 [hep-th]].

[2] S. Ryu and T. Takayanagi, "Holographic derivation of entanglement entropy from AdS/CFT, " Phys. Rev. Lett. 96, 181602 (2006) hep-th/0603001.

[3] L. Susskind, "Computational Complexity and Black Hole Horizons, " arXiv:1402.5674 [hep-th].

[4] M. Miyaji, T. Numasawa, N. Shiba, T. Takayanagi and K. Watanabe, "Gravity Dual of Quantum Information Metric," arXiv:1507.07555 [hep-th].

[5] A. Uhlmann, "The transition probability in the state space of A*-algebra," Rep. Math. Phys. 9273 (1976),

[6] S-J. Gu, "Fidelity Approach to Quantum Phase Transi- tions," Int. J. Mod. Phys. B 24 (2010) 4371 arXiv:0811.3127 [quant-ph]].

[7] M. Alishahiha, "Holographic Complexity," Phys. Rev. D 92, no. 12, 126009 (2015) arXiv:1509.06614 [hep-th]].

[8] M. Henningson and K. Skenderis, "The Holographic Weyl anomaly," JHEP 9807, 023 (1998) hep-th/9806087.

[9] L. Y. Hung, R. C. Myers and M. Smolkin, "Some Calculable Contributions to Holographic Entanglement Entropy," JHEP 1108, 039 (2011) arXiv:1105.6055 [hep- th]].

[10] M. Mir, "On Holographic Weyl Anomaly," JHEP 1310, 084 (2013) [arXiv:1307.5514 [hep-th]].

[11] D. Carmi, R. C. Myers and P. Rath, "Comments on Holographic Complexity," JHEP 1703, 118 (2017) arXiv:1612.00433 [hep-th]].

[12] C. R. Graham and E. Witten, "Conformal anomaly of submanifold observables in AdS / CFT correspondence," Nucl. Phys. B 546, 52 (1999) hep-th/9901021.

[13] S. Kachru, X. Liu and M. Mulligan, "Gravity duals of Lifshitz-like fixed points," Phys. Rev. D 78, 106005 (2008) arXiv:0808.1725 [hep-th]].

[14] S. Banerjee, J. Erdmenger and D. Sarkar, "Connecting Fisher information to bulk entanglement in holography," arXiv:1701.02319 [hep-th].

[15] D. Momeni, A. Myrzakul, R. Myrzakulov, S. Alsaleh and L. Alasfar, "Thermodynamics of AdS spacetime via Regularized Fidelity Susceptibility," arXiv:1704.05785] [hepth].

[16] J. D. Brown, J. Creighton and R. B. Mann, "Temperature, energy and heat capacity of asymptotically antide Sitter black holes," Phys. Rev. D 50, 6394 (1994) gr-qc/9405007. 